\title{
Correlation between Diameter of the Pampiniform Plexus in Millimeter Measured by Scrotal Doppler Ultrasound with the Seminal Fluid Parameters
}

\author{
Muhsin Alqasar ${ }^{1}$ and Azzawi M Hadi*2 \\ ${ }^{1}$ Department of Surgery, Mosul University College of Medicine, Iraq \\ ${ }^{2}$ Department of Surgery, Tikrit University College of Medicine, Iraq \\ *Corresponding author: Azzawi M Hadi, Department of Surgery, Tikrit University College of Medicine, Iraq
}

Submission: 海 May 24, 2018; Published: 海 August 20, 2018

\begin{abstract}
The study involves 158 infertile persons suffering from primary or secondary infertility. This study aimed to fine the correlation between diameter of pampiniform plexus veins measured by scrotal Doppler ultrasound with parameters of seminal fluid analysis. There was negative correlation between diameter of pampiniform plexus veins and the concentration of sperms/ml, TPFS, sperm normal morphology, and the active sperm movement, while the correlation was positive the abnormal sperms, sluggishly moved, and non motile sperms, this correlations were statistically significant. According to this study, the seminal fluid parameters are correlated significantly with the diameter the spermatic cord veins measured by Doppler ultrasound.
\end{abstract}

Keywords: Seminal fluid; Correlation; Varicocele; Doppler ultrasound

\section{Introduction}

The dilation and tortuosity of the pampiniform plexus known as varicocele, which present in $15 \%$ of the male population, and in approximately $40 \%$ of men presenting with infertility [1]. Testicular temperature elevation and venous reflux appear to play an important role in varicocele- induced testicular dysfunction, although the exact pathology of varicocele induced damage is not yet completely understood [2-4]. In a study of 200 men with varicocele, a mean sperm density of less than 20x106 sperm/ml, and low motility in $90 \%$ of patients, a shift of sperm shape from oval to tapered and amorphous, and an increase in immature sperm forms [5]. Total potentially functional spermatozoa (TPFS)-an index of total count multiplied by percentage of actively motile sperms multiplied by normal morphology-is affected deleteriously by varicocele [5]. Varicocele was considered significant when it has a diameter of $3 \mathrm{~mm}$ or greater and retrograde flow on Doppler ultrasound examination. The reversal flow characteristic of varicocele is confirmed by prolonged flow augmentation within a flow area depicted as reversing (i.e. changing color) on real-time imaging. Two or more tortuous, sonolucent tubular structure with a caliber of $3 \mathrm{~mm}$ or more are seen as the hallmark finding in varicoceles [6]. Color Doppler imaging has a sensitivity of $93 \%$ in detection of of venographically diagnosed varicoceles as compared with 71\% using physical examination [7]. Ultrasound was found to have a $95 \%$ sensitivity in the detection of subclinical varicoceles [8]. The aim of the study is to correlate between the diameter of the pampiniform plexus and seminal fluid parameter.

\section{Patients and Methods}

The study involves 158 patient with history of primary or secondary infertility (patient with azoospermia were excluded). Referred to the urology clinic in Tikrit teaching hospital and private clinic between January 2006 to January 2008. Evaluation of patients includes detailed history, physical examination, seminal fluid analysis, and colored Doppler study. Doppler study done for measurement of the diameter of the veins of the pampiniform plexus (with valsalva maneuver) in millimeters. Statistical analysis and correlation between the results of Doppler study and the parameters of seminal fluid parameters was done by using Person's formula.

\section{Results}

Patients were aged between 20-64 years old, 93 (59\%) of them suffered primary infertility and 65(41\%) had secondary infertility with a duration of infertility ranged from 1-21 year. As seen in Table 1 , the diameter of both right and left pampiniform plexus veins had negative correlation with concentration, total potentially functioning sperms, normal morphology and active motile sperms. This correlation was statistically significant $(p<0.05)$. In contrast, the correlation was positive for both right and left pampiniform plexus with abnormal morphology, sluggishly and non motile sperms this correlation was statistically significant $(\mathrm{p}<0.05)$. Total potentially functioning sperms, normal morphology, and concentration had the more negative correlation $(-0.292,-0.273$, and -0.266 respective- 
ly) with the diameter of right plexus veins than the correlation with left vein diameter with the same variables these were $(-0.249,-0.27$, and -0.226 respectively). Non motile and sluggishly motile sperms had more positive correlation with right $(0.285$, and 0.272 respec- tively) than with the left pampiniform plexus diameter $(0.22$, and 0.269 respectively). The abnormal morphology had more positive correlation with the left plexus than the right $(0.253$, and 0.209 respectively).

Table 1: Correlation between the diameters of pampiniform plexus measured by scrotal Doppler ultrasound with the parameters of the seminal fluid analysis (concentration, total potential functioning sperms, morphology and motility).

\begin{tabular}{|c|c|c|c|c|c|c|c|}
\hline \multirow{2}{*}{ Conc. } & \multirow{2}{*}{ TPFS } & \multicolumn{2}{|c|}{ Morphology } & \multicolumn{2}{c|}{ Motility } \\
\cline { 3 - 8 } & & & Normal & Abnormal & Active & Sluggish & Non \\
\hline Rt. vein & -0.266 & -0.292 & -0.273 & 0.209 & -0.2 & 0.272 & 0.285 \\
\hline Lt. vein & -0.226 & -0.249 & -0.27 & 0.253 & -0.279 & 0.269 & 0.22 \\
\hline
\end{tabular}

\section{Discussion}

In this study we didn't take the varicocele as a variable in the study instead we analyze the diameter of the veins and this include the normal and all the grade of the varicocele. There was a weak but significant negative correlation with concentration, total potential active sperms, highly active sperms, and normal morphology, while the correlation was positive (though weak but significant) with the sluggish and non motile sperms as well as abnormal sperms. This correlation can be explained by the deleterious effect of the dilated veins on the sperm count, morphology and activity [5]. Many studies had been published concerning the role of the varicocele in male infertility, but the issue is still controversial. The exact action mechanism of the varicocele's negative effect on sperm function and fertility is not resolved [9]. A study conducted by the WHO in 1992 showed a decrease in testicular volume, and total sperm per ejaculate in those with varicocele, however; the other sperm characteristics of motility and morphology were not influenced by the presence of varicocele [10]. Other study showed that all sperm parameters were found to be significantly lower in patient with varicocele than fertile subject without varicocele, but no correlation was found with the varicocele grade [11] Much of the human data studying the underlying mechanisms of varicocelerelated infertility are derived by measuring parameters in men with and without varicocele and between men pre and post varico celectomy. However, none of these associations is able clearly to explain the variable effect of varicocele or directly explain the mechanism(s) involved, especially for the bilateral effect. The etiology may be multi factorial, and may even include a preexisting genetic disposition, which may account for variable effect of varicocele on spermatogenesis and infertility seen in human [1]. There is no doubt that large scale, carefully designed and controlled studies must be conducted to elucidate the role of varicocele in infertility, and to clarify which sperm function test are associated with reduced fertility potential so that those who may benefit from correction of varicocele can be identified [9].

\section{References}

1. Cathy KN, Ajay KN, Ashok A (2005) Pathophysiology of varicocele in male infertility. Hum Reprod Update 7(5): 473-481.

2. Klaiber EL, Broverman DM, Pokoly TB, Albert AJ, Howard PJ, et al. (1987) Interrelationships of cigarette smoking, testicular varicocele, and seminal fluid indexes. Fertil Steril 47(3): 481-486.

3. Sigman M, Jarow JP (1997) Ipsilateral testicular hypotrophy is associated decreased sperm count in infertile men with varicocele. J Urol 158(2): 605-607.

4. Yamamoto M, Hibi H, Katsuno S, Miyake K (1995) Effect of varicocelectomy on testis volume and semen parameters in adolescents: a randomized prospective study. Nagoya J Med Sci 58(3-4): 127-132.

5. Hauster R, Botchan A, Yogev L, Yavetz H (2001) Varicocele and human infertility; effect of varicocele on sperm function. Hum Reprod Update 7(5): 482-485.

6. Gonda RL, Karo JJ, Forte RA, O’Donnell KT (1997) Diagnosis of subclinical varicocele in infertility. Am J Roentgenol 148(1): 71-75.

7. Nashan D, Behre HM, Grunert JH, Nieschlag E (1990) Diagnostic value of scrotal sonography in infertile men: report on 658 cases. Andrologia 22(5): 387-395.

8. Patel PJ, Pareek S (1989) Scrotal ultrasound in male infertility. Eur Urol 16(6): 423-425.

9. Pierik FH, Vreeburg JT, Stijnen TH, van Roijen JH, Dohle GR, et al. (1998) Improvement of sperm count and motility after ligation of varicocele detected by colored Doppler ultrasonography. Int J Androl 21(5): 256-260.

10. World Health Organization (1992) The influence of varicocele on parameters of fertility in a large group of men presenting to infertility clinics. Fertil Steril 57(6): 1289-1293.

11. Villnueva DC, Vega HE, Diaz PM, Echavarria Sanchez M, Karchmer Krivitsky S (1999) Sperm dysfunction in subfertile patients with varicocele and marginal semen analysis; Andrologia 31(5): 263-267. 


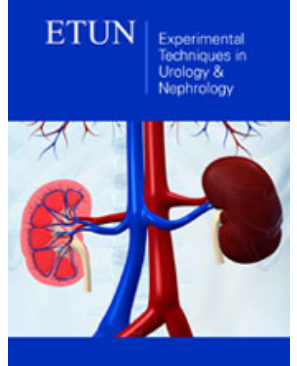

Experimental Techniques in Urology \& Nephrology Benefits of Publishing with us

- High-level peer review and editorial services

- Freely accessible online immediately upon publication

- Authors retain the copyright to their work

- Licensing it under a Creative Commons license

- Visibility through different online platforms 